\title{
Ear, Nose and Throat Examination in Epidermolysis Bullosa and Procedural Approach
}

\author{
Ismail Cem Temel ${ }^{1}$, Asli Bilgic ${ }^{2}$ and Dedee Frances Murrell*2,3 \\ ${ }^{1}$ Head and Neck Surgery, Chris O'Brien Life House, Sydney, Australia \\ ${ }^{2}$ Department of Dermatology, St George Hospital, UNSW, Sydney, Australia \\ ${ }^{3}$ Faculty of Medicine, University of New South Wales, Sydney, Australia
}

Received: 制: October 4, 2018; Published: 制: October 10, 2018

*Corresponding author: Dedee Frances Murrell, Prof. and Head of Department of Dermatology, St George Hospital, UNSW, Sydney, NSW, Australia

\begin{abstract}
Epidermolysis Bullosa is a spectrum of genetic diseases containing more than 25 subtypes of diseases. The most important characteristic of this disease group is the predisposition towards mechanical fragility which results in blistering of skin and mucous membranes. Mucosal involvement of the ear, nose, and throat areas can cause significant morbidity, especially in junctional and recessive dystrophic EB subtypes. ENT surgeons are frequently involved in the management of EB patients. Thus, in this article, we summarize the important points for ENT surgeons in the management, examination and procedural approach for EB patients.
\end{abstract}

Keywords: Epidermolysis Bullosa; Ear, Nose and Throat; Mucosal Involvement; Management; Surgical Procedures

Abbreviations: EB: Epidermolysis Bullosa; EBS: Epidermolysis Bullosa simplex; JEB: Junctional Epidermolysis Bullosa; DEB: Dystrophic Epidermolysis Bullosa; ENT: Ear, nose, and throat

\section{Introduction}

Epidermolysis Bullosa (EB) is a spectrum of genetic bullous diseases containing more than 25 subtypes of bullous diseases. The pathogenesis involves genetic defects in structural proteins of the skin and mucosa. The most important characteristic of this disease group is the predisposition towards mechanical fragility which results in blistering of the skin and mucous membranes. Mucosal involvement including ear, nose, and throat (ENT) areas can be significant, especially in severe subtypes such as junctional EB (JEB) and recessive dystrophic EB (RDEB) [1]. However, even with the mild EB types like EB simplex (EBS), ENT procedures involved in examination and/or management of EB patients might lead discomfort, blistering, bleeding and erosions on the mucosae. Thus, ENT surgeons should apply specific examination and procedural approach in the care of these patients. In this article, we aimed to summarise general recommendations and approaches to ENT examination and procedures in EB.

\section{Ear, Nose, and Throat Involvement}

ENT involvement is commonly seen in JEB and recessive DEB in which different structural proteins found in dermoepidermal junction and/or dermis are defective. However, mild forms (EBS) might also cause problems which complicate these patients' lives and reduce their quality of life. Although EB usually starts at birth, mild cases can go unnoticed. Symptoms can change according to the affected area and severity of the involvement.

Involvement of the oral mucosa usually causes pain, bleeding because of erosions. Epistaxis and nose obstruction can be experienced with nasal mucosal involvement. Otitis media and otitis externa can be frequent in patients with EB [2-4]. However, most problematic mucous involvement areas are pharynx (causing dysphagia, odynophagia) and larynx (causing dysphonia, inspiratory stridor, hoarse cry, exuberant granulation tissue, scarring of the vocal cords, laryngeal stenosis and/or stricture). These areas often affected by more severe EB types (JEB and recessive DEB) [2-4]. All patients with EB should also be evaluated by a dentist to identify any problem because dental and mucosa abnormalities, enamel hypoplasia, frequent caries is common especially in recessive DEB and JEB [1].

\section{Procedural Approach}

Patients with EB can need routine ENT procedures for diagnosis of related and unrelated problems and/or therapeutic approaches. The most common surgical procedure within the ENT in severe EB patients is tracheostomy placement. Others surgical procedures includes unrelated surgeries like tonsillectomy, adenoidectomy and other surgical procedures within the ears or nasal cavity [2]. Safe 
and effective procedural approach is essential. Albeit, there are no established guidelines for management of non-invasive or invasive procedures in patients with EB. Often, EB patients are managed in multidisciplinary specialised units. However, in case of need every ENT surgeon should be aware of some of the basic steps in management of such patients [5-8].

a. Patients should carry on their usual skin care habits like dressings, emollients and topical therapies during inpatient units before and after surgery.

b. Preoperative assessment of practitioners (ENT surgeon and/or anesthetist) who will be involved in the procedure is necessary

c. EB subtype and involvement of mucous area should be known before planning any procedure

d. The presence of other systemic complications (anaemia, renal or cardiac problems which are common in EB) should also be taken into account. Moreover, anaemia and malnutrition should be treated as they slow down the healing process of EB lesions.

e. All topical and systemic therapies (even intermittent ones such as steroids) used for the patients should be known and noted in the file

f. Routine anesthesia procedures carry greater risks than general population. Thus, all possible techniques (local/ regional anesthesia with/without sedation, inhalation/ intravenous general anesthesia, endotracheal intubation/ laryngeal mask airway) should be discussed and management should be personalized.

g. If patient had a history of same or similar procedure and/ or anesthesia, files for the procedure should be reviewed for any complication happened previously

h. Anticipated risks about the procedure and anesthesia should be explained to patients and/or care givers

i. Protection of skin and mucous membrane integrity is essential. In every procedure, practitioners should be careful not to create mechanical trauma like direct pressure and/or friction on surfaces.

j. For this reason, avoid rigid endoscopes and nasogastric tubes

k. Lubrication of necessary equipment like endoscopes, laryngoscopes, endotracheal and nasogastric tubes is in great importance to minimize the trauma.

l. Avoid applying adhesive tapes used for pulse oximeter and temperature probes, ECG and electrocautery electrodes directly to the skin

m. Use of clip-on pulse oximeter probes n. If not available, use pulse oximeter probes and intravenous cannulas with polyvinyl chloride film covers

o. Avoid using plastic oxygen masks which are hard and/or with sharp edges

p. Often EB patients are children. Thus, sedative premedication before any anesthesia type might be useful

q. For monitoring blood pressure during surgery/procedure, PVC film or cotton padding can be used to reduce the friction

r. Practitioners should be aware of EB patients having increased risk of fluid and heat loss during surgeries and there is increased risk of secondary infection, pain and scarring formation after surgery

s. Difficult intubation can be seen in severe types such as JEB and recessive DEB because of limited mouth opening. Thus, endotracheal intubation is often preferred as it is secure. When positioning any intubation apparatus, skin and mucous membranes should be protected from damage with lubricated gauze. Also, before extubating mucous membranes should be checked.

t. Dexamethasone $(0.25 \mathrm{mg} / \mathrm{kg})$ can be given to reduce airway swelling postoperatively if it has been a difficult intubation.

u. Allocation of enough time to surgery is important as operations usually require more time than usual.

v. Eye protection is also an important issue during surgery. Thus, they should be closed and covered. However, adhesive tapes and eye ointments are not recommended.

w. Careful transportation is necessary before and after surgery to minimize trauma.

x. Postoperative analgesia is essential as pain can cause irritation of the patient and can result in more skin damage.

y. New blisters should be drained by releasing the fluid using a sterile needle. Leaving the overlying skin intact is often recommended to provide a biological dressing [5-8].

\section{Conclusion}

The ENT specialist should be aware of the skin and mucous fragility of patients with EB and try to provide a safe and effective procedural approach during mucosal examination and/or procedures.

\section{Acknowledgement}

Dr. Ismail Cem Temel is a consultant ENT surgeon at Seydişehir State Hospital, Konya, Turkey. He is currently working as an observer at Head and Neck Surgery Unit, Chris O’Brien Lifehouse, Sydney, Australia.

Dr. Asli Bilgic is a consultant dermatologist and now working as a Visiting Fellow at St. George Hospital, UNSW. She is the recipient 
of Turkish Dermatology Society - Prof. Dr. Hulusi Behcet (Longterm Research) Scholarship in 2018.

\section{Funding}

This article did not receive any specific grant from funding agencies in the public, commercial, or not-for-profit sectors for publication.

\section{References}

1. Fine JD, Bruckner Tuderman L, Eady RA, Bauer EA, Bauer JW, et al. (2014) Inherited epidermolysis bullosa: updated recommendations on diagnosis and classification. J Am Acad Dermatol 70(6): 1103-1126.

2. Fine JD, Johnson LB, Weiner M, Suchindran C (2007) Tracheolaryngeal complications of inherited epidermolysis bullosa: cumulative experience of the national epidermolysis bullosa registry. Laryngoscope. 117(9): 1652-1660.

3. Fine JD, Mellerio JE (2009) Extracutaneous manifestations and complications of inherited epidermolysis bullosa: part II. Other organs. J Am Acad Dermatol 61(3): 387-402.

\section{ISSN: 2574-1241}

DOI: $10.26717 / B J S T R .2018 .09 .001866$

Dedee Frances Murrell. Biomed J Sci \& Tech Res

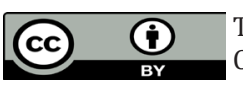

This work is licensed under Creative Commons Attribution 4.0 License

Submission Link: https://biomedres.us/submit-manuscript.php
4. Wright JT (2010) Oral manifestations in the epidermolysis bullosa spectrum. Dermatol Clin 28(1): 159-164.

5. Nandi R, Howard R (2010) Anesthesia and epidermolysis bullosa. Dermatol Clin 28(2): 319-324.

6. Palinko D, Matievics V, Szegesdi I, Sztano B, Rovo L (2017) Minimally invasive endoscopic treatment for pediatric combined high-grade stenosis as a laryngeal manifestation of epidermolysis bullosa. Int J Pediatr Otorhinolaryngol 92: 126-129.

7. Haruyama T, Furukawa M, Matsumoto F, Kawano K, Ikeda K (2009) Laryngeal stenosis in epidermolysis bullosa dystrophica. Auris Nasus Larynx 36(1): 106-109.

8. Hore I, Bajaj Y, Denyer J, Martinez AE, Mellerio JE, et al. (2007) The management of general and disease specific ENT problems in children with Epidermolysis Bullosa--a retrospective case note review. Int J Pediatr Otorhinolaryngol 71(3): 385-391.

$\begin{array}{ll}\text { BIOMEDICAL } & \text { Assets of Publishing with us } \\ \text { RESEARCHES } & \text { - Global archiving of articles } \\ \text { - Immediate, unrestricted online access } & \text { - Rigorous Peer Review Process } \\ \text { https://biomedres.us/ }\end{array}$

